\title{
Auswahlermessen der Zulassungsgremien bei der Neubesetzung einer Vertragsarztzulassung im Fachgebiet Radiologie
}

\section{Rechtliche Vorgaben}

Verzichtet ein Vertragsarzt auf seine Vertragsarztzulassung zum Zwecke der Nachbesetzung, so regelt § 103 Abs. 4 S. 5 SGB V die Kriterien, auf deren Grundlage die Zulassungsgremien die Auswahl zwischen mehreren Bewerbern zu treffen haben. Dies sind:

- die berufliche Eignung,

- das Approbationsalter,

- die Dauer der ärztlichen Tätigkeit,

- eine mindestens fünf Jahre dauernde vertragsärztliche Tätigkeit in einem Gebiet, in dem der Landesausschuss nach § 100 Abs. 1 SGB V das Bestehen von Unterversorgung festgestellt hat,

- ob der Bewerber Ehegatte, Lebenspartner oder ein Kind des bisherigen Vertragsarztes ist,

- ob der Bewerber ein angestellter Arzt des bisherigen Vertragsarztes oder ein Vertragsarzt ist, mit dem die Praxis bisher gemeinschaftlich betrieben wurde,

- ob der Bewerber bereit ist, besondere Versorgungsbedürfnisse, die in der Ausschreibung der Kassenärztlichen Vereinigung definiert worden sind, zu erfüllen,

- Belange von Menschen mit Behinderung beim Zugang zur Versorgung,

- bei medizinischen Versorgungszentren die Ergänzung des besonderen Versorgungsangebots; dies gilt entsprechend für Vertragsärzte und Berufsausübungsgemeinschaften mit einem besonderen Versorgungsangebot.

Der Standort, an dem die Versorgung erfolgen soll, spielt in dieser Aufzählung keine Rolle, spezielle Versorgungsgesichtspunkte eher am Rande (siebtes und neuntes Kriterium). Dies liegt darin begründet, dass $§ 103$ Abs. 4 S. 5 SGB V die Nachbesetzung einer bereits an einem bestimmten Ort vorhandenen Vertragsarztzulassung regelt. Abweichend davon gestaltet sich die Rechtslage dann, wenn eine Vertragsarztzulassung aufgrund einer (partiellen) Entsperrung eines Planungsbereichs neu besetzt werden soll. Hier regelt §26 Abs. 4 Nr. 3 Bedarfspla-
nungsrichtlinien-Ärzte, dass weitere Kriterien von den Zulassungsgremien zu prüfen sind, nämlich

- die bestmögliche Versorgung der Versicherten im Hinblick auf die räumliche Wahl des Vertragsarztsitzes,

- sowie Versorgungsgesichtspunkte; z. B. Fachgebietsschwerpunkt, Feststellungen nach $\S 35$ BedarfsplanungsrichtlinienÄrzte (z. B. Förderung bestimmter Versorgungsangebote).

Im Rahmen dieser Prüfung geht es also nicht darum, eine vorhandene Vertragsarztzulassung mit dem bestgeeigneten Bewerber im Rahmen der vorhandenen Praxisstruktur nach zu besetzen, sondern primär gestalterisch auf die Versorgung der Versicherten einzuwirken.

Welche Anforderungen sind insofern an den Sachvortrag der Bewerber zu stellen, inwieweit müssen die Zulassungsgremien Sachverhaltsermittlung betreiben, um ihr Auswahlermessen rechtssicher ausüben zu können und welche Kriterien kommen im Rahmen der Ausübung eines solchen gestalterischen Ermessens in Betracht? Dass diese Fragen nicht einfach zu beantworten sind, ergibt sich bereits daraus, dass die Sozialgerichte sich in mehreren Entscheidungen mit diesen Fragen befassen mussten und das Auswahlermessen der Zulassungsgremien als unzureichend ausgeübt beanstandet haben. Ein Blick in die einschlägige diesbezügliche Rechtsprechung der Sozialgerichte erscheint daher lohnenswert.

\section{Wohnortnahe Versorgung}

In dem der Entscheidung des Sozialgerichts Marburg vom 16.03.2016, Aktenzeichen S 12 KA 170/15, zu Grunde liegenden Fall bewarben sich insgesamt zwölf Radiologinnen und Radiologen auf eine, durch partielle Öffnung eines Planungsbereichs neu zu besetzende, Vertragsarztzulassung im Fachgebiet Radiologie. Das Sozialgericht Marburg hat zunächst klargestellt, dass die Schaffung größerer Planungsbereiche im Rahmen der Bedarfsplanung für das Fach-

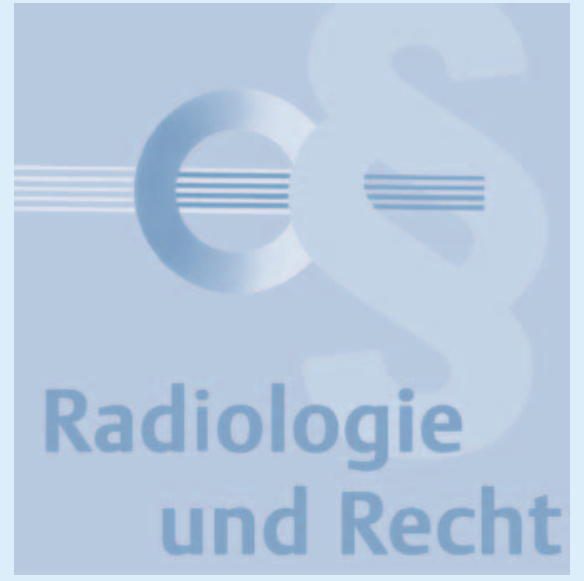

gebiet Radiologie und die Berücksichtigung von - lokalen - Versorgungsgesichtspunkten keinen Widerspruch darstellen. Der Gesetzgeber habe ausweislich des Gesetzes zur Verbesserung der Versorgungsstrukturen in der gesetzlichen Krankenversicherung (GKV-Versorgungsstrukturgesetz vom 20.12.2011, BGBI I 2011, 2983) die Sicherstellung einer flächendeckenden bedarfsgerechten und wohnortnahen medizinischen Versorgung der Bevölkerung nicht aufgeben wollen. Hieran knüpfe die Regelung in § 26 Abs. 4 Nr. 3 BedarfsplanungsRichtlinien-Ärzte an. Daher seien bei der partiellen Öffnung von Planungsbereichen die von den Bewerbern auf die neu zu besetzende Vertragsarztzulassung genannten Standorte nicht gleich zu gewichten; ein Kriterium für die Neubesetzung einer derartigen Vertragszulassung sei auch, welcher Standort am ehesten die wohnortnahe medizinische Versorgung der Bevölkerung gewährleiste. Das hat zur Konsequenz, dass Zulassungsgremien - ähnlich wie bei Sonderbedarfszulassungen - den Bedarf in der Umgebung des von dem jeweiligen Bewerber genannten Standorts ermitteln müssen. Zwar kommt dem Zulassungsausschuss bei der Beurteilung eines solchen Versorgungsbedarfs ein Ermessensspielraum zu. Die ordnungsgemäße Ausübung dieses Ermessens setzt aber voraus, dass die Zulassungsgremien die für die Ausübung des Ermessens relevanten Anknüpfungstatsachen vollständig ermittelt haben.

Hierzu gehört nicht nur die Erstellung einer Versorgungsanalyse, aus der sich ergibt, wie viele Einwohner der Einzugsbereich des von dem jeweiligen Bewerber genannten 
Standortes hat, sondern auch, inwieweit in diesem Einzugsbereich gehäuft - als Indiz für eine unzureichende Versorgung - Kostenübernahmeanträge gegenüber den gesetzlichen Krankenkassen gestellt werden. Ermittelt werden muss weiterhin, in welchem Umfang Versicherte im Einzugsbereich des von dem jeweiligen Bewerber geplanten Standorts im Rahmen einer Ermächtigung (im Fall des Sozialgerichts Marburg für die Spezialisierung Kinderradiologie) versorgt werden. Die Zulassungsgremien müssen sich im Rahmen ihrer Ermessensausübung damit auseinandersetzen, ob nicht dem Radiologen, der über eine solche Ermächtigung verfügt und der sich auf die neu zu besetzende Vertragsarztzulassung bewirbt, der Vorrang vor den anderen Bewerbern einzuräumen ist. Bei der Feststellung der bereits vorhandenen radiologischen Versorgung ist nicht nur zu berücksichtigen, wie viele Versorgungsaufträge im Einzugsbereich des von dem jeweiligen Bewerber genannten Standorts anfallen, sondern auch, in welchem Umfang bereits radiologische Leistungen im Rahmen einer dort angesiedelten Nebenbetriebsstätte erbracht werden.

\section{Spezielle Versorgungs- gesichtspunkte}

Im oben genannten Fall des Sozialgerichts Marburg war von einem der Bewerber geltend gemacht worden, die Vorhaltung eines offenen MRT sei ein besonderer, zu berücksichtigender Versorgungsgesichtspunkt. Dieser besondere Versorgungsgesichtspunkt sei auch deshalb zu berücksichtigen, weil er aufgrund der Vorhaltung des offenen MRT viele Angstpatienten habe, was sich auch in der Zahl der Kostenübernahmeanträge gegenüber den gesetzlichen Krankenkassen niederschlage.

Das Sozialgericht Marburg hat zunächst in Frage gestellt, ob dem gesetzlich Versicherten grundsätzlich einen Anspruch auf Versorgung mit einem offenen MRT zusteht, wenn in vertretbarer Entfernung ein geschlossenes MRT zur Versorgung zur Verfügung steht. Die Möglichkeit, besondere Patientengruppen mittels offenem MRT behandeln zu können, sei jedenfalls keine fachliche Spezialisierung im Sinne eines besonderen Versorgungsgesichtspunkts, sondern lediglich eine apparative Modernisierung. Dies komme schon darin zum Ausdruck, dass Ärzte eine besonders vereinbarte Qualifikation zur Abrechnung einer mittels offenem MRT durchgeführten Behandlung nicht benötigten und die Diagnostik im EBM nicht gesondert ausgewiesen sei. Daraus folge, dass das offene MRT eine Behandlungsvariante sei, die für die Patienten, insbesondere diejenigen mit klaustrophobischen Einschränkungen, schonender und komfortabler sei; dies könne jedoch nicht als besonderer Versorgungsgesichtspunkt im Rahmen der Auswahl auf eine neu zu besetzende Vertragsarztzulassung berücksichtigt werden; schließlich bestehe die Möglichkeit einer den Patienten im Regelfall zumutbaren Sedierung.

Das Sozialgericht München hat dies in dem der Entscheidung vom 07.11.2018, Aktenzeichen S 38 KA 634/17, zu Grunde liegenden Fall anders gewichtet. Es gehe bei dem Einsatz eines offenen MRT nicht allein um eine schonendere und komfortablere Versorgungsart. Aus Sicht des Sozialgerichts München ist ein offenes MRT eine Versorgungsbesonderheit, die im Rahmen des Auswahlermessens der Zulassungsgremien bei Neubesetzung einer Vertragsarztzulassung zu berücksichtigen sei. Allerdings reiche hierfür der Hinweis, ein offenes MRT zu betreiben, nicht aus. Vielmehr müsse das Vorhandensein von offenen MRT im Planungsbereich quantifiziert, eine Schätzung des Bedarfs abgegeben und dargestellt werden, welcher Anteil an MRT-Untersuchungen insgesamt auf Untersuchungen am offenen MRT entfällt. Insofern müsse zusätzlich gewichtet werden, ob diese Untersuchungen am offenen MRT tatsächlich bestimmten Patientengruppen zugutekämen oder schlicht ersatzweise anstelle eines geschlossenen MRT erfolgten.

Das Sozialgericht München hat sich in dieser Entscheidung noch mit der Relevanz weiterer Versorgungsgesichtspunkte befasst.

So könne eine „exzentrische“ Lage, die durch das Fehlen einer wohnortnahen radiologischen Versorgung gekennzeichnet sei, im Rahmen des Auswahlermessens nicht besonders berücksichtigt werden. Von einer „exzentrischen“ Lage sei auszugehen, wenn der von dem jeweiligen
Bewerber genannte Standort sich z. B. in einer Grenzlage befinde und nach einer Richtung ohne Hinterland sei (was bislang andere Radiologen davon abgehalten hat, dort eine Praxis zu betreiben). Eine solche „exzentrische“ Lage sei deshalb nicht als geeigneter Standort für eine neue Vertragsarztzulassung zu präferieren, da eine solche Auswahlentscheidung mit dem Gebot der gleichmäßigen Verteilung der Vertragsarztsitze in einem großräumigen Planungsbereich kaum zu vereinbaren wäre.

Ob in der Tätigkeit eines Programmverantwortlichen Arztes im Mammografie Screening ein bei der Auswahl auf eine neu zu besetzende Vertragsarztzulassung zu berücksichtigendes Alleinstellungsmerkmal zu sehen ist, war ebenfalls Gegenstand der Erwägungen der Zulassungsgremien und gleichzeitig Anlass für eine Prüfung durch das Sozialgericht, ob das Auswahlermessen durch die Zulassungsgremien zutreffend ausgeübt worden ist. Der Inhaber des PVAVersorgungsauftrages hatte argumentiert, der PVA-Versorgungsauftrag ergebe deshalb ein im Rahmen des Auswahlermessens zu berücksichtigendes Alleinstellungsmerkmal, weil die Erreichbarkeit der ScreeningEinheit wesentlich darüber entscheide, wie hoch die Abbruchquote im Rahmen der Abklärung unklarer Befunde sei. Diese sei in seinem Screening-Bereich mit 60 Prozent vergleichsweise hoch, was eben daran liege, dass die Abklärung stationär in einer Praxis erfolgen müsse, nicht mobil, und eine solche Praxis wohnortnah nicht existiere; aus diesem Grund erstrebe er seine Auswahl auf die neu zu besetzende Vertragsarztzulassung für diesen Standort.

Im Urteil vom 24.01.2018, Aktenzeichen S 38 KA 971/16, hatte sich das Sozialgericht München noch kritisch dazu geäußert, dass sich aus der PVA-Tätigkeit eines Bewerbers ein Alleinstellungsmerkmal ergeben soll. Es sei kaum möglich, eine Kausalität zwischen Standort und Abbrecherquote nachzuweisen. Mit dem Urteil vom 07.11.2018, Aktenzeichen S 38 KA 634/17, hat sich das Sozialgericht München dahingehend selbst relativiert. Es sei zu berücksichtigen, dass die Tätigkeit des programmverantwortlichen Arztes in regelmäßigen Abständen einer Rezertifizierung zu unterziehen sei. Ferner erfahre die Tätigkeit als programmverantwortlicher Arzt dadurch eine überre- 
gionale Bedeutung, dass Statistiken erstellt und die Daten bundesweit ausgewertet werden müssten. Sollte die Rezertifizierung deshalb infrage stehen und damit die Ziele der Krebsfrüherkennungsrichtlinie gefährdet werden, weil die Voraussetzungen für eine Rezertifizierung aufgrund fehlender Zulassung an einem bestimmten Standort (z. B. Nichterreichen der notwendigen Klientinnenzahl) nicht erfüllt werden können, so käme einem Bewerber auf eine neu zu besetzende Vertragsarztzulassung, der zugleich PVA ist, ein besonderer Stellenwert im Rahmen der zu treffenden Auswahlentscheidung zu. Dies sei allerdings konkret nachzuweisen.

Schließlich hat sich das Sozialgericht München noch mit der Frage befasst, welche Auswirkungen die Tätigkeit eines Bewerbers als Honorarkonsul auf die Auswahlentscheidung der Zulassungsgremien hat.
Nach Art. 5 des „Wiener Übereinkommens für konsularische Beziehungen “ stünden im Vordergrund der Tätigkeit als Honorarkonsul der Schutz der Interessen des Entsendestaates und seiner Angehörigen sowie die Förderung der Entwicklung der Beziehungen auf dem Gebiet des Handels, der Wirtschaft, des Fremdenverkehrs, der Kultur und der Wissenschaft. Die Interessen des Entsendestaates sind aber bei der an dem Bedarf der gesetzlich Versicherten in einem Planungsbereich auszurichtenden Auswahlentscheidung der Zulassungsgremien nicht zu berücksichtigen, so dass sich aus dieser Tätigkeit kein Alleinstellungsmerkmal ergibt.

\section{Fazit}

Bewerbungen auf durch eine (partielle) Öffnung von Planungsbezirken geschaffene
Vertragsarztzulassungen bedürfen sorgfältiger Vorbereitung; die reine stichwortartige Behauptung von speziellen Versorgungsgesichtspunkten hilft in der Regel nicht weiter. Andererseits setzt die Ausübung des Auswahlermessens seitens der Zulassungsgremien eine sorgfältige Aufbereitung der Auswahlkriterien voraus. Rechtsbehelfe gegen Auswahlentscheidungen der Zulassungsgremien können daher in den Fällen erfolgversprechend sein, in denen abgelehnten Bewerbern der Nachweis gelingt, dass die Zulassungsgremien „nicht genau genug hingesehen“ haben.

Dr. Horst Bonvie
Rechtsanwalt
Fachanwalt für Medizinrecht
Rechtsanwälte Wigge
Großer Burstah 42
20457 Hamburg

